\title{
Identification of hydroxy-plastochromanol in Arabidopsis leaves
}

\author{
Renata Szymańska and Jerzy Kruk ${ }^{凶}$ \\ Department of Plant Physiology and Biochemistry, Faculty of Biochemistry, Biophysics and Biotechnology, Jagiellonian University, Kraków, Poland
}

\begin{abstract}
In the present study we have identified hydroxy-plastochromanol in plants for the first time. This compound was found both in low light and high light-grown Arabidopsis plants, however, under high light stress its level was considerably increased. Hydroxy-plastochromanol accumulated also during ageing of leaves of low lightgrown plants, similarly as in the case of other prenyllipids. Our results indicate that hydroxy-plastochromanol found in leaves is probably formed as a result of plastochromanol oxidation by singlet oxygen generated in photosystem II during photosynthesis. These data also support the hypothesis that plastochromanol is an efficient antioxidant in vivo, similarly as tocopherols and plastoquinol.
\end{abstract}

Keywords: Arabidopsis, hydroxy-plastochromanol, plastochromanol, plastoquinol, tocopherol, light stress

Received: 26 November, 2009; revised: 15 February, 2010; accepted:12 March, 2010; available on-line: 16 March, 2010

\section{INTRODUCTION}

Plastochromanol (PC) is a naturally occurring $\gamma$-tocotrienol homologue with a longer side chain of eight isoprenoid units, which is synthesized from a reduced plastoquinone by tocopherol cyclase (Kumar et al., 2005; Szymańska \& Kruk, 2010; Zbierzak et al., 2010), the key enzyme in tocopherol biosynthesis. PC was first identified as a minor component in leaves of rubber tree (Whittle et al., 1965) and later in leaves of other plant species (Dunphy et al., 1966; Strzałka et al., 2009). It is found at the highest amounts in flax and rape seed oils (Balz et al., 1992; Gofmann \& Möllers, 2000; Velasco \& Goffman, 2000; Kalique et al., 2005; Gruszka \& Kruk, 2007), as well as in minor amounts in seed oils of camelina (Zubr \& Matthäus 2002; Gruszka \& Kruk, 2007), maize (Gruszka \& Kruk, 2007), hemp (Kriese et al., 2004), many Vietnamese plants (Matthäus et al., 2003), salvia (Bagci \& Karaagacli, 2004), black pine (Bagci et al., 2004) and others (Gruszka \& Kruk, 2007). Its function in seeds seems to be similar to that of tocopherols, i.e., antioxidant action but the literature data on that subject is very limited (Olejnik et al., 1997). Recently, PC has been found in substantial amounts in Arabidopsis leaves and seeds (Szymańska \& Kruk, 2010; Zbierzak et al., 2010) and it has been suggested to play there the role of an antioxidant (Szymańska \& Kruk, 2010). On the other hand, it has been shown that PC is an efficient singlet oxygen quencher in vitro, being even more active than $\gamma$-tocopherol or $\gamma$-tocotrienol in hydrophobic solvents (Gruszka et al., 2008). In the process of singlet oxygen quenching, not only the aromatic ring of plastochromanol is active but also the long unsaturated side-chain. It is known that compounds containing conjugated double bonds, like carotenoids, are very efficient singlet oxygen quenchers (Di Mascio et al., 1990). Even molecules with an isolated double bonds can be efficient quenchers, especially if the double bond is at a tertiary carbon atom (Bonesi et al., 2006), like in prenyllipids with an unsaturated side chain (Gruszka et al., 2008). In contrast to tocopherols and tocotrienols, the main scavenging products of plastochromanol were monohydroxy- (PC-OH) and trihydroxy-derivatives (Gruszka et al., 2008), although 8-hydroperoxy- $\gamma$-tocopherones of all these compounds were also found.

If $\mathrm{PC}$ is a singlet oxygen scavenger in vivo, its hydroxyl-derivatives should be detectable in leaves. Until now, there are no reports in the literature on the occurrence of such derivatives. In the present study we analyzed Arabidopis leaves grown under different light intensity conditions for oxidation products of plastochromanol.

\section{MATERIALS AND METHODS}

Plant material and growth conditions. Arabidopsis thaliana plants (wild-type Col-0, vte4 and vte1 mutants) were grown under low light conditions $\left(100 \mu \mathrm{mol} \mathrm{m} \mathrm{m}^{-2}\right.$ $\mathrm{s}^{-1}, 22^{\circ} \mathrm{C}, 12 / 12 \mathrm{~h}$ light/dark period) as well as high light conditions $\left(380 \mu \mathrm{mol} \mathrm{m}-2 \mathrm{~s}^{-1}, 26^{\circ} \mathrm{C} / 17^{\circ} \mathrm{C}, 12 / 12 \mathrm{~h}\right.$ light/dark period). Strong light was applied by $400 \mathrm{~W}$ metal halide lamps (Kolorarc, Hungary) with double thermal glass filters to minimize overheating of plants. The higher temperature during the photoperiod of high light conditions was a consequence of heat emission from the light source even though the heat filters were used. The average temperature of high light conditions corresponds to low light conditions and therefore we do not expect any significant influence of these differences on the composition of prenyllipids.

The vte1 mutant (GABI_111D07, line 2) carries TDNA insertion in intron 3 of the At4g32770 gene, while the vte4 mutant (SALK_036736) shows T-DNA insertion in intron 1 of the At1g64970 gene (Mène-Saffrané \& DellaPenna, 2009).

For analysis, 4-5 week-old plants were used, unless otherwise stated. The old and young leaves were analyzed separately.

Prenyllipid analysis. For quantitative analysis of prenyllipids, three Arabidopsis leaves were ground in a mortar with $1.5 \mathrm{ml}$ of cold ethyl acetate. Then, $400 \mu \mathrm{l}$

هe-mail: jerzy.kruk@uj.edu.pl

Abbreviations: $\mathrm{HL}$, high light; $\mathrm{LL}$, low light; $\mathrm{PC}$, plastochromanol; $\mathrm{PC}-\mathrm{OH}$, hydroxy-plastochromanol; $\mathrm{PQ}-9$, plastoquinone; $\mathrm{PQH}_{2}-9$, plastoquinol; Toc, tocopherol; WT, wild type. 
of the extract was transferred to an Eppendorf tube and evaporated under nitrogen. The evaporated extract was dissolved in HPLC solvent (methanol/hexane, $340: 20, \mathrm{v} / \mathrm{v}$ ), centrifuged shortly on a benchtop centrifuge $(10000 \mathrm{~g} \times 10 \mathrm{~s})$ and analyzed immediately. The HPLC measurements were performed using a $100 \mu \mathrm{l}$ loop, Jasco PU-980 pump and UV-VIS detector system UV-970 connected in line with a Shimadzu RF10AXL fluorescence detector (excitation/emission detection at 290/330 nm), a Teknokroma (Barcelona, Spain) $\mathrm{C}_{18}$ reverse-phase column (Nucleosil 100, $250 \mathrm{~mm} \times 4$ $\mathrm{mm}, 5 \mu \mathrm{m})$, isocratic solvent system - methanol/hexane $(340: 20, \mathrm{v} / \mathrm{v})$ at the flow rate of $1.5 \mathrm{ml} / \mathrm{min}$. Oxidized PQ was followed by absorbance at $255 \mathrm{~nm}$ while other prenyllipids were monitored using fluorescence detection as described in detail by Kruk and Karpinski (2006). Taking into account that plastochromanol and its hydroxyl-derivatives have the same chromanol ring structure (chromophore) as that of $\gamma$-tocopherol, for quantitative HPLC analysis the response of the fluorescence detector, using excitation at $290 \mathrm{~nm}$ and emission detection at $330 \mathrm{~nm}$, was taken to be the same for both these compounds.

For preparative analysis, ethyl acetate extracts from 10 rosettes were pooled and separated on a Nucleosil column (as above) in methanol at the flow rate of $1.5 \mathrm{ml} /$ $\mathrm{min}$ and the peak corresponding to $\mathrm{PC}-\mathrm{OH}$ was collected.

Absorbance spectra were recorded on a Varian Cary 400 Bio UV-VIS spectrophotometer.

Mass spectrometry analysis was performed using an Esquire 3000 mass spectrometer (Bruker Daltonics, Bremen, Germany) equipped with a standard electrospray ion source. The prenyllipid was dissolved in a solution of methanol/formic acid (99.9:0.1, v/v). Samples were introduced into the ion source using a syringe pump (KD Scientific, USA, model KDS100) with the flow rate set to 3 microliters per minute. Voltage between capillaries was set to $4500 \mathrm{~V}$, temperature of heated capillary to $280^{\circ} \mathrm{C}$. Additional parameters (voltages on skimmer 1, skimmer 2, octopole, trap drive potential, etc.) were optimized according to the scan range used during analyses.

Prenyllipid standards. Tocopherol homologues of HPLC grade $(\geq 99.5 \%)$ were purchased from Merck. Oxidized and reduced plastoquinone standards were obtained as described by Kruk (1988). The plastochromanol standard was purified from linseed oil according to the published procedure (Gruszka et al., 2008) or synthesized from natural PQ as described by Gruszka and Kruk (2007).

\section{RESULTS}

In the HPLC chromatogram of leaf extracts of Arabidopsis wild type (WT), using fluorescence detection for tocochromanols and reduced prenyllipids, apart from peaks of the known compounds, an additional peak was found with the retention time of $6 \mathrm{~min}$ (Fig. 1). The compound corresponding to this peak was also present in the vte 4 mutant lacking $\alpha$-tocopherol ( $\alpha$-Toc) but it was not found for the vte 1 mutant lacking all tocopherols and plastochromanol. This indicates that this peak could be a PC derivative. The subsequent preparative isolation of this compound and spectroscopic analysis showed that it has identical absorption spectra to those of PC and $\gamma$-Toc. The absorbance maxima were at $294.5 \mathrm{~nm}$ and $300.5 \mathrm{~nm}$ with a minimum at about $260 \mathrm{~nm}$ both in cyclohexane

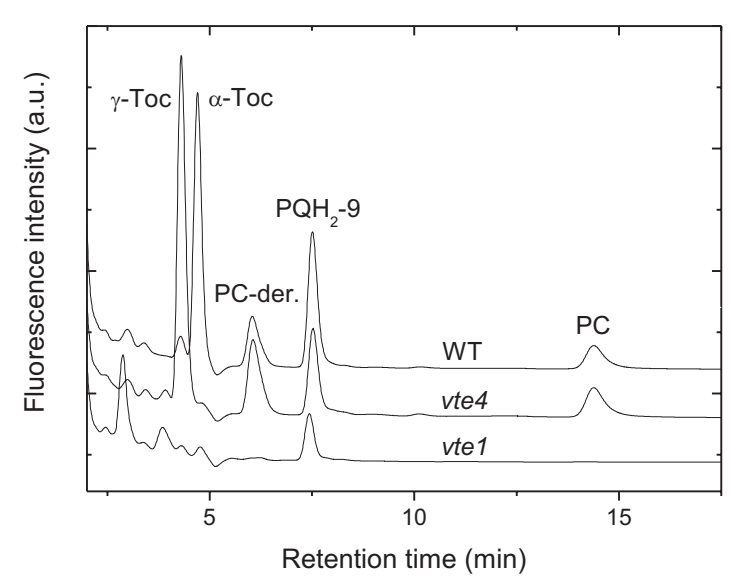

Figure 1. HPLC chromatograms of leaf extracts from Arabidopsis

The extracts from wild type (WT) and tocopherol biosynthetic mutants (vte4, vte1) were analyzed using fluorescence detection (ex./ em. $=290 / 330$ nm). PC-der., PC-derivative.

and iso-octane. The obtained absorbance maxima of the investigated compound are the same as those reported for PC and $\gamma$-Toc in the literature (Whittle et al., 1965; Thies, 1997). This indicates that all three compounds have the same structure of the chromanol ring. The considerably shorter retention time of the investigated compound
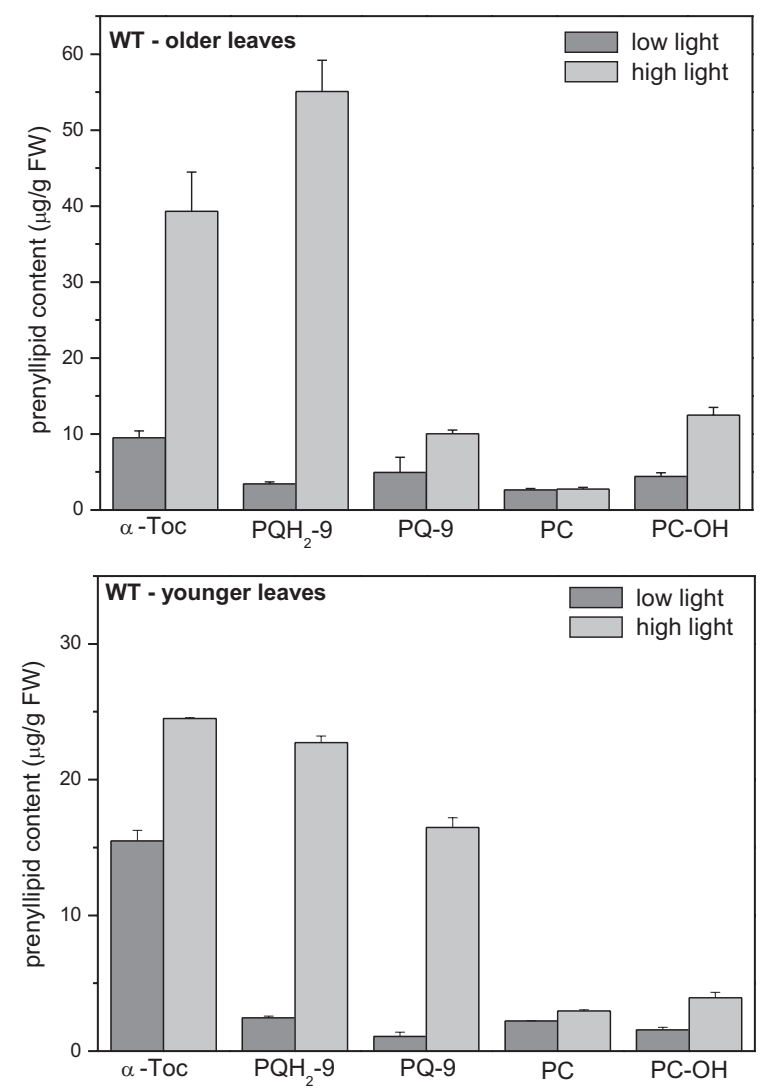

Figure 2. Prenyllipid content in older and younger leaves of Arabidopsis

Four- to five-week-old WT plants grown under low and high light conditions were analyzed. Data are means \pm S.E. $(n=3-5)$. 

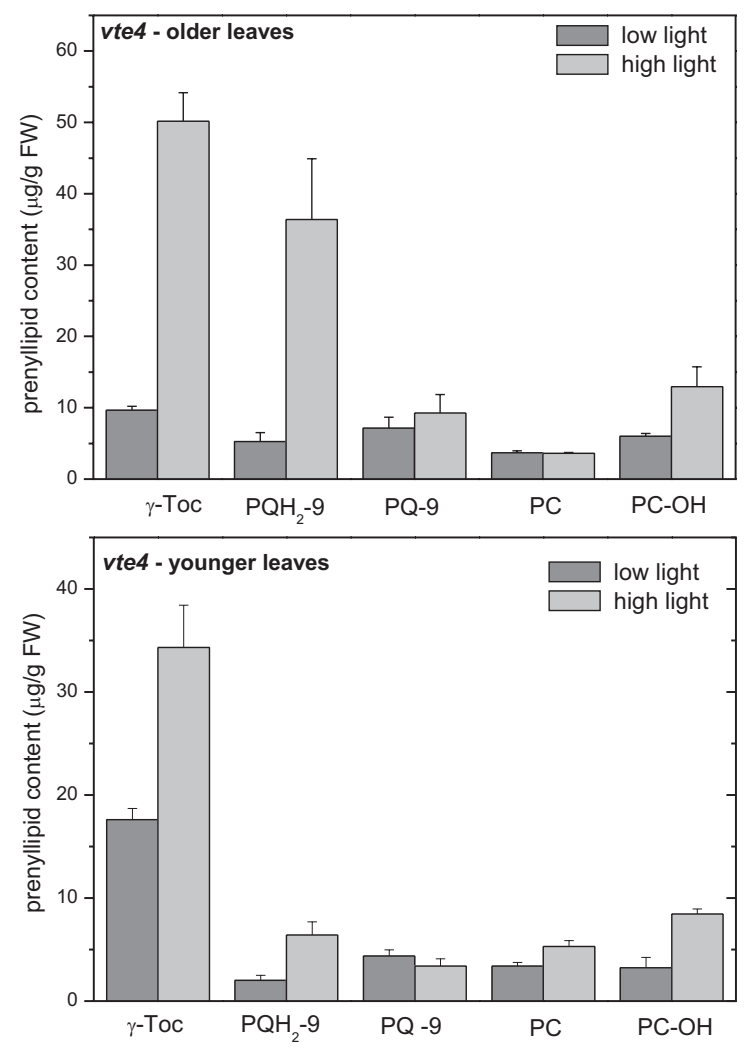

Figure 3. Prenyllipid content in older and younger leaves of Arabidopsis

Four- to five-week-old plants of a-Toc-deficient mutant vte4 grown under low and high light conditions were analyzed. Data are means \pm S.E. $(n=3-5)$.

as compared to PC, together with the identical spectroscopic properties, indicates that the new compound is a hydroxyl-derivative of PC with the hydroxyl group(s) located in the side-chain. A mass spectrometry analysis of this compound showed a $\mathrm{m} / \mathrm{z}=806.0$ corresponding to $[\mathrm{PC}+\mathrm{O}+\mathrm{K}]^{+}$ion, which is in agreement with the molecular mass of a potassium adduct of PC having one hydroxyl group (PC-OH). An analogous peak for the oxidation product of $\mathrm{PC}$ by singlet oxygen was observed in vitro (Gruszka et al., 2008). Observation of the PC-OH pseudomolecular ion as an adduct with potassium most probably results from the high affinity of hydroxylated PC for potassium ions originating from the glass vessels used for sample preparation.

Figure 2 shows that high light stress stimulates strongly the synthesis of $\alpha$-Toc and of plastoquinol $\left(\mathrm{PQH}_{2}-9\right)$ both in older and younger leaves of the WT, while the level of PC remains relatively low under both light conditions. In the older leaves of low-light-grown plants, the PC-OH level is slightly higher than the level of PC but nearly four-fold higher under high light conditions. In the younger leaves, the content of both compounds was similar.

In the vte 4 mutant lacking $\alpha$-Toc, the level of $\gamma$-Toc is similar to that of $\alpha$-Toc in the WT (Fig. 3). The response of all the prenyllipids to high light is similar to that observed for the WT plants when the older leaves are concerned, while in the case of the younger leaves the increase in the level of both PQ forms is less pronounced.

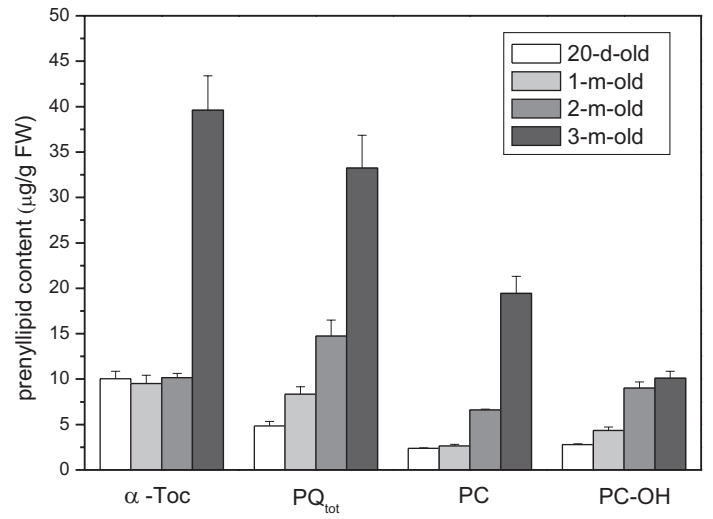

Figure 4. Prenyllipid content in leaves of Arabidopsis in the course of aging

The WT plants grown under low-light conditions were analyzed. Data are means \pm S.E. $(n=3-5)$.

When the content of prenyllipids in leaves of WT plants grown under low light conditions was analyzed in the course of aging (Fig. 4), the content of both PC and $\mathrm{PC}-\mathrm{OH}$ increased. When 2- and 3-month old leaves were compared, the level of $\mathrm{PC}-\mathrm{OH}$ was similar, while in the case of PC it increased several-fold. Within the analyzed growth period, the total level of PC and PC$\mathrm{OH}$ was similar to that of total (oxidized and reduced) PQ.

In seeds of the WT, PC-OH was not detected in contrast to PC which amounts to $10 \%$ of all the prenyllipids (Szymańska \& Kruk, 2010).

\section{DISCUSSION}

The obtained results demonstrate a strong increase in the levels of tocopherols and plastoquinol, the known lipophilic antioxidants, under high light conditions, which is supposed to be the response of Arabidopsis plants to oxidative stress. It has been already shown that massive accumulation of total plastoquinone (mainly plastoquinol) takes place at high light conditions in sun-exposed leaves of beech, as compared to shade leaves (Lichtenthaler 1971; 1977; 2007). However, the accumulation of $\alpha$-tocopherol was less pronounced in those studies than in our case. It was also found that in high light-grown Arabidopsis plants the level of $\alpha$-tocopherol increased several-fold as compared to low light-grown plants (Golan et al., 2006). In a recent paper of Zbierzak et al. (2010) it was shown that the level of PC increased over 3-fold under high-light stress applied for 4 days, which is in contrast to our results. However, in our experiments the plants were acclimated to high light conditions for a long period of growth and during this time some of the $\mathrm{PC}$ formed was probably converted to $\mathrm{PC}-\mathrm{OH}$, whose level clearly increased under high light conditions.

In the present study we have identified for the first time hydroxyl-plastochromanol in plants. Regarding the position of the hydroxyl group in the molecule, there is a question of the order of the carbon atom and the position of the isoprene unit where the group is attached. If the hydroxyl group is formed due to the oxidation of a double bond in the side chain by singlet oxygen, and the mechanism of the side chain oxidation is the same as that of the double bond in cholesterol (Gruszka et 
al., 2008), then the hydroxyl group will be of the second order. Taking into account that the oxidation is a nonenzymatic reaction, theoretically all the double bonds in the PC side chain could be oxidized and a mixture of isomers formed. This could be the case for the PC-OH isolated in our study. The HPLC system applied would be probably unable to resolve individual isomers differing in the position of the hydroxyl group at different isoprene units.

Since PC-OH has been identified in vitro as the main oxidation product of PC during singlet oxygen scavenging (Gruszka et al., 2008), our results indicate that such a reaction possibly also occurs in vivo and indicates that the main function of PC in leaves is quenching and scavenging of singlet oxygen generated in photosystem II during high light stress, similarly as in the case of tocopherol and plastoquinol (Krieger-Liszkay, 2004; Kruk et al., 2005; Kruk \& Trebst, 2008). The absence of PC-OH in seeds of Arabidopsis, where no formation of singlet oxygen is expected, also supports this hypothesis. The origin of $\mathrm{PC}-\mathrm{OH}$ as the oxidation product of $\mathrm{PC}$ by singlet oxygen or by other reactive oxygen forms, is also indicated by its increased level in leaves of Arabidopsis grown under high light conditions. Nevertheless, $\mathrm{PC}-\mathrm{OH}$ is also formed under low light conditions, where the formation of reactive oxygen forms is expected to be considerably less pronounced. It also cannot be excluded that $\mathrm{PC}-\mathrm{OH}$ is formed enzymatically in leaves from PC to some extent by a still unidentified oxidase. It is also possible that $\mathrm{PC}-\mathrm{OH}$ has some other metabolic function. It should be also pointed out that $\mathrm{PC}-\mathrm{OH}$ itself can be potentially a singlet oxygen scavenger because of the presence of the chromanol ring. Experiments on the singlet oxidation of PC in vitro (Gruszka et al., 2008) showed that besides the hydroxylated products of PC, also PC-OH homologue of 8 -hydroperoxy- $\gamma$-tocopherone was formed showing that this compound is a singlet oxygen scavenger at least in the investigated system. It is also worth mentioning that also other hydroxylated derivatives of prenyllipids, such as plastoquinone- $\mathrm{C}$, have been detected in higher plants and algae (Kruk \& Strzałka, 1998; Kruk \& Trebst, 2008) and could be products of the singlet oxygen scavenging activity of prenyllipids (Gruszka et al., 2008). However, in the case of Arabidopsis, only trace amounts of plastoquinone-C were detected (Kruk, unpublished).

\section{Acknowledgements}

This work was supported by grant N302 04932 from the Ministry of Science and Higher Education.

\section{REFERENCES}

Ahmed MK, Daun JK, Przybylski R (2005) FT-IR based methodology for quantitation of total tocopherols, tocotrienols and plastochromanol-8 in vegetable oils. I Food Compos Anal 18: 359-364.

Bagci E, Karaagacli Y (2004) Fatty acid and tocochromanol patterns of Turkish pines. Acta Biol Cracov Bot 46: 95-100.

Bagci E, Vural M, Dirmenci T, Bruehl L, Aitzetmuller K (2004) Fatty acid and tocochromanol patterns of some Salvia L. species. Z Naturforsch 59c: 305-309.

Balz M, Schulte E, Thier HP (1992) Trennung von Tocopherolen und Tocotrienolen durch HPLC. Fat Sci Technol 94: 209-213.

Bonesi SM, Fagnoni M, Monti S, Albini A (2006) Reaction of singlet oxygen with some benzylic sulfides. Tetrabedron 62: 10716-10723.

Di Mascio P, Devasagayam TPA, Kaiser S, Sies H (1990) Carotenoids, tocopherols and thiols as biological singlet molecular oxygen quenchers. Biochem Soc Trans 18: 1054-1056.
Dunphy PJ, Whittle KJ, Pennock JF (1966) Plastochromanol. In Biochemistry of Chloroplasts; vol 1; Goodwin TW ed, pp 165-171. Academic Press, London.

Goffman FD, Möllers C (2000) Changes in tocopherol and plastochromanol-8 contents in seeds and oil of oilseed rape (Brassica napus L.) during storage as influenced by temperature and air oxygen. J Agric Food Chem 48: 1605-1609.

Golan T, Muller-Moule P, Niyogi KK (2006) Photoprotection mutants of Arabidopsis thaliana acclimate to high light by increasing photosynthesis and specific antioxidants. Plant Cell Environ 29: 879-887.

Gruszka J, Kruk J (2007) RP-LC for determination of plastochromanol, tocotrienols and tocopherols in plant oils. Chromatographia 66: 909-913.

Gruszka J, Pawlak A, Kruk J (2008) Tocochromanols, plastoquinol, and other biological prenyllipids as singlet oxygen quenchers-determination of singlet oxygen quenching rate constants and oxidation products. Free Radic Biol Med 45: 920-928.

Krieger-Liszkay A (2004) Singlet oxygen production in photosynthesis. $J$ Exp Bot 56: 337-346.

Kriese U, Schumann E, Weber WE, Beyer M, Brühl L, Matthäus B (2004) Oil content, tocopherol composition and fatty acid pattern of the seeds of 51 Cannabis sativa L. genotypes. Euphytica 137: 339351.

Kruk J (1988) Charge-transfer complexes of plastoquinone and $\alpha$-tocopherol quinone in vitro. Biophys Chem 30: 143-149.

Kruk J, Strzałka K (1998) Identification of plastoquinone-C in spinach and maple leaves by reverse-phase high-performance liquid chromatography. Phytochemistry 49: 2267-2271.

Kruk J, Karpinski S (2006) An HPLC-based method of estimation of the total redox state of plastoquinone in chloroplast, the size of the photochemically active plastoquinone-pool and its redox state in thylakoids of Arabidopsis. Biochim Biophys Acta 1757: 1669-1675.

Kruk J, Trebst A (2008) Plastoquinol as a singlet oxygen scavenger in photosystem II. Biochim Biophys Acta 1777: 154-162.

Kruk J, Holländer-Czytko H, Oettmeier W, Trebst A (2005) Tocopherol as singlet oxygen scavenger in photosystem II. I Plant Physiol 162: 749-757.

Kumar R, Raclaru M, Schüßeler T, Gruber J, Sadre R, Lühs W, Zarhloul KM, Frierdt W, Enders D, Frentzen M, Weier D (2005) Characterisation of plant tocopherol cyclases and their overexpression in transgenic Brassica napus seeds. FEBS Lett 579: 1357-1364.

Lichtenthaler HK (1971) Die unterschiedliche Synthese der lipophilen Plastidenchinone in Sonnen und Schattenblättern von Fagus silvatica L. $Z$ Naturforsch 26b: 832-842.

Lichtenthaler HK (1977) Regulation of prenylquinone synthesis in higher plants. In Lipids and Lipid Polymers in Higher Plants. Tevini M, Lichtenthaler HK, eds, pp 231-258. Springer, Berlin.

Lichtenthaler HK (2007) Biosynthesis, accumulation and emission of carotenoids, tocopherol, pastoquinone and isoprene in leaves under high photosynthetic irradiance. Photosynth Res 92: 163-179.

Matthaus B, Vosmann K, Pham LQ, Aitzetmüller K (2003) FA and tocopherol composition of Vietnamese oilseeds. I Am Oil Chem Soc 80: 1013-1020.

Mène-Saffrané L., DellaPenna D (2009) Biosynthesis, regulation and functions of tocochromanols in plants. Plant Physiol Biochem, doi:10.1016/j.plaphy.2009.11.004.

Olejnik D, Gogolewski M, Nogala-Kaucka M (1997) Isolation and some properties of plastochromanol-8. Nabrung 41: 101-104.

Strzałka K, Szymańska R, Świeżewska E, Skorupińska-Tudek K, Suwalsky M (2009) Tocochromanols, plastoquinone and polyprenols in selected plant species from Chilean Patagonia. Acta Biol Cracov Bot 51: $39-44$.

Szymańska R, Kruk J (2010) Plastoquinol is the main prenyllipid synthesized during acclimation to high light conditions in Arabidopsis and is converted to plastochromanol by tocopherol cyclase. Plant Cell Physiol, doi: $10.1093 / \mathrm{pcp} / \mathrm{pcq} 017$.

Thies W (1997) Entwiclung von Ausgangsmaterial mit erhöhten aoder $\gamma$-Tocopherol-Gehalten im Samenöl für die Körnerraps-Züchtung. Angew Bot 71: 62-67.

Whittle KJ, Dunphy PJ, Pennock JF (1965) Plastochromanol in the leaves of Hevea brasiliensis. Biochem J 96: 17c-19c.

Velasco L, Goffman FD (2000) Tocopherol, plastochromanol and fatty acid patterns in the genus Linum. Plant Syst Evol 221: 77-88.

Zbierzak AM, Kanwischer M, Wille C, Vidi PA, Giavalisco P, Lohmann A, Briesen I, Porfirova S, Brehelin C, Kessler F, Dormann P (2010) Intersection of the tocopherol and plastoquinol metabolic pathways at the plastoglobule. Biochem J 425: 389-399.

Zubr J, Matthäus B (2002) Effects of growth conditions on fatty acids and tocopherols in Camelina sativa oil. Ind Crop Prod 15: 155-162. 